\title{
VitisCyc: a metabolic pathway knowledgebase for grapevine (Vitis vinifera)
}

\section{Sushma Naithani ${ }^{1}{ }^{*}$, Rajani Raja ${ }^{1}$, Elijah N. Waddell' ${ }^{1}$, Justin Elser ${ }^{1}$, Satyanarayana Gouthu ${ }^{2}$, Laurent G. Deluc ${ }^{2}$ and Pankaj Jaiswal ${ }^{1}$}

Department of Botany and Plant Pathology, Oregon State University, Corvallis, OR, USA

2 Department of Horticulture, Oregon State University, Corvallis, OR, USA

\section{Edited by:}

Mario Pezzotti, University of

Verona, Italy

Reviewed by:

Abdullahil Baque, Sher-e-Bangla Agricultural University, Bangladesh Marianna Fasoli, University of Verona, Italy

Jerome Grimplet, Instituto de Ciencias de la Vid y del Vino, Spain

*Correspondence:

Sushma Naithani, Department of Botany and Plant Pathology, Oregon State University, 2082 Cordley Hall, Corvallis, OR-97331, USA e-mail:naithans@science. oregonstate.edu
We have developed VitisCyc, a grapevine-specific metabolic pathway database that allows researchers to (i) search and browse the database for its various components such as metabolic pathways, reactions, compounds, genes and proteins, (ii) compare grapevine metabolic networks with other publicly available plant metabolic networks, and (iii) upload, visualize and analyze high-throughput data such as transcriptomes, proteomes, metabolomes etc. using OMICs-Viewer tool. VitisCyc is based on the genome sequence of the nearly homozygous genotype PN40024 of Vitis vinifera "Pinot Noir" cultivar with 12X v1 annotations and was built on BioCyc platform using Pathway Tools software and MetaCyc reference database. Furthermore, VitisCyc was enriched for plant-specific pathways and grape-specific metabolites, reactions and pathways. Currently VitisCyc harbors 68 super pathways, 362 biosynthesis pathways, 118 catabolic pathways, 5 detoxification pathways, 36 energy related pathways and 6 transport pathways, 10,908 enzymes, 2912 enzymatic reactions, 31 transport reactions and 2024 compounds. VitisCyc, as a community resource, can aid in the discovery of candidate genes and pathways that are regulated during plant growth and development, and in response to biotic and abiotic stress signals generated from a plant's immediate environment. VitisCyc version 3.18 is available online at http://pathways.cgrb.oregonstate.edu.

Keywords: grape, VitisCyc, grapevine pathway database, Vitis vinifera, microarray

\section{INTRODUCTION}

Grapevine (Vitis spp) is one of the most economically important and widely cultivated fruit crops in the world, covering $\sim 8$ million hectares and producing $\sim 67.5$ million tons of berries (http://www.oiv.int/). The majority of grape berries are processed into wine, but significant portions of the harvest provide fresh table grapes, raisins, juice, vinegar, and distilled spirits (http:// faostat.fao.org/). In addition to its economic importance, grapes are rich in nutrients, metabolites, vitamins, volatile compounds, and dietary fibers-all of which offer potential benefits to human health (De et al., 2010; Smoliga et al., 2011; Wu and Hsieh, 2011).

The large-scale genomic datasets are of tremendous value in extending the knowledge of grape metabolism, scoring cultivar specific differences, and understanding how the plant's overall metabolism is affected by diseases and abiotic stress conditions resulting from changes in the photoperiod, temperature, moisture, climate etc. The current availability of genomics reasources for grape include genome sequence of two genotypes PN40024 and ENTAV 115 of the Vitis vinifera "Pinot Noir" cultivar (Jaillon et al., 2007; Velasco et al., 2007), and Vitis9kSNP array based genome diversity data for $950 \mathrm{~V}$. vinifera and $59 \mathrm{~V}$. sylvestris accessions (Myles et al., 2011). In addition, more than 50 varieties of grapevine are being sequenced at least at 10X coverage (www.vitaceae.org/index.php/Current_Sequencing_Projects) to probe the genetic diversity in the cultivated and wild grape accessions. Genetic linkage maps are available for various cultivars of V. vinifera (Doligez et al., 2002; Vezzulli et al., 2008) and quantitative trait loci (QTLs) have been identified for several traits including seedlessness, fungal disease resistance, and fruit yield components (Doligez et al., 2002; Fischer et al., 2004; Costantini et al., 2008; Salmaso et al., 2008). In addition to ESTs (Da Silva et al., 2005), microarrays and RNA-Seq data sets (Terrier et al., 2005; Waters et al., 2005, 2006; Espinoza et al., 2006; Cramer et al., 2007; Deluc et al., 2007, 2009; Fernandez et al., 2007; Grimplet et al., 2007; Pilati et al., 2007; Tattersall et al., 2007; Chervin et al., 2008; Figueiredo et al., 2008; Gatto et al., 2008; Lund et al., 2008; Mathiason et al., 2009; Zamboni et al., 2010; Zenoni et al., 2010), a few metabolomes and proteomes of grape berry have been published (Sarry et al., 2004; Giribaldi et al., 2007; Martinez-Esteso et al., 2011; Dai et al., 2013; Martínez-Esteso et al., 2013).

The lack of a comprehensive, evidence-based, grapevinespecific cellular metabolic framework limits the analysis and full utilization of the genomic scale expression data. We created VitisCyc, a metabolic pathway database, based on the genome sequence of the Vitis vinifera "Pinot Noir" accession PN40024, using $12 \mathrm{X}$ v1 annotations, provided by Centro di Ricerca Interdipartimentale per le Biotechnologie Innovative (CRIBI) (http://www.cribi.unipd.it/). VitisCyc was built on widely used BioCyc platform (Karp and Caspi, 2011; Karp et al., 2013) 
and contains metabolic and transport pathways, enzymes, transporters, genes, metabolites, and small biomolecules that are either experimentally determined or predicted to be present within a grapevine cell. The database is supported by extensive manual curation, and it facilitates incremental integration of existing and new information.

Grapes have been analyzed extensively for secondary metabolites related to color, taste, aroma etc. using chemical, physiological, and metabolic approaches. Thus, it provides an excellent opportunity for evidence-based curation of secondary metabolic compounds, reactions and pathways. At present, our curation efforts in VitisCyc are heavily focused on the secondary metabolic pathways. The knowledge derived from grape could be of use for discovering similar pathways in other fleshy fruits and to lesser extent for enriching model plant pathway databases, such as AraCyc (Arabidopsis thaliana) (Zhang et al., 2005), MedicCyc (Medicago truncatula) (Urbanczyk-Wochniak and Sumner, 2007), RiceCyc (Oryza sativa, rice) (Dharmawardhana et al., 2013), and MaizeCyc (Zea mays, maize) (Monaco et al., 2013) etc., which significantly lack curation of secondary metabolic pathways.

VitisCyc allows users to search or browse for pathways, reactions, enzymes, genes, and compounds as well as supports analysis of user-defined, large-scale expression data in context of the overall cellular metabolic network using the OMICs-Viewer tool. VitisCyc can be used for developing cell, tissue or organ specific metabolic models and/or simulations representing changes in the grapevine cellular metabolic networks under various treatments or environmental stresses. In this publication, we report the development and curation of the VitisCyc database version 3.18 and show utility of OMICs-Viewer tool by analyzing expression data from pulp tissue of grape berries representing four ripening stages.

\section{MATERIALS AND METHODS CONSTRUCTION OF VitisCyc Genome, proteins, and annotation methods}

VitisCyc was constructed based on the genome sequence of grape cultivar "Pinot Noir" (genotype PN40024) with $12 \mathrm{X}$ v1 annotations provided by CRIBI (http:/genomes.cribi.unipd.it/ grape/) that represents 29,971 protein coding genes. We used the Pathways Tools software (Karp et al., 2002) to create VitisCyc by following the instructions of the software developers on the input data file contents and formats. Accordingly, PRODUCTTYPE code "P" was assigned for a protein or a hypothetical ORF. Each gene/protein record includes mandatory attributes: NAME (gene/protein name), and FUNCTION (name of the enzyme), and recommended attributes: Locus ID or gene ID, Enzyme Commission (EC) number and Gene Ontology (GO) assignments. The optional attributes include SYNONYM or gene symbols, and COMMENT (a free text description). Only the FUNCTION, SYNONYM, EC number, DBLINK and GO attributes have multiple values. The older grape GeneIDs from the 8X (Jaillon et al., 2007) and 12X v0 version (Adam-Blondon et al., 2011) were stored as SYNONYMS. SYNONYMS also include names and synonyms imported from the homologous $A$. thaliana and $O$. sativa gene entries using Inparanoid-based approach used previously for genome annotations of $F$. vesca (Shulaev et al.,
2011) and E. grandis (Myburg et al., 2014). The FUNCTION values were extracted from the GO annotations derived from the IntrerproScan, Interpro2GO and EC2GO pipelines developed by Jaiswal laboratory that have been used for development of RiceCyc (Dharmawardhana et al., 2013) and MaizeCyc (Monaco et al., 2013).

\section{Mapping of the reactions and pathways}

After collecting the annotations for grape proteins in the ATTRIBUTE-VALUE standard format, and appropriately writing the GENETIC ELEMENT (required) and FASTA sequence files (optional), the "PathoLogic" option was executed (with taxonomic filtering on) to find best matches for grape proteins in the reference database MetaCyc. When a match was found for an entity, such as, an enzyme name, gene name, synonym, EC number, reaction etc., then the respective reaction and pathway were projected in the VitisCyc.

\section{Quality control filters}

To ensure consistency and accuracy, a quality control check was performed after initial assembly of VitisCyc, following the standard procedure of the Pathway Tools suite (Karp et al., 2002) with taxonomic filtering on. Subsequently, enrichment of plant pathways in the VitisCyc were done by comparing it with PlantCyc (version 5.0), a plant-specific metabolic network (Chae et al., 2012). If, different names for the same protein were found across various sources, then enzyme names were manually edited to match with associated EC and GO terms, while other names were included as FUNCTION-SYNONYM attributes for EC/GO match. The pathways specific to bacteria, fungi and animals that bypassed the taxonomic filtering were removed. In some cases, enrichment and manual curation of name-based assignments were also done. The PathoLogic tool provides a list of potential name matches and EC numbers (if available), along with the reactions. Often automated assignments fail due to change in the official enzyme names or due to assignment of new EC numbers, or no EC number for an enzyme. The annotations assigned due to partial or incomplete domain presence were also excluded. However, such errors are flagged alerting the curators to manually edit the list and make recommendations to confirm/edit/reject the existing reaction(s) or to create new reactions. Additional checks included visual confirmation of domains (InterPro) prior to final updates and rescoring of pathways. Pathway Tool software is updated routinely, whenever a new version becomes available, the current version is 18.0. Also, pathways and reactions are rescored and crosschecked twice per year against the new release of the manually curated gold standard reference libraries provided by the MetaCyc and PlantCyc.

\section{GENE EXPRESSION ANALYSES USING OMICs-VIEWER TOOL}

To show functionality of OMICs-Viewer tool, we used a subset of microarray expression data (NCBI GEO accession \#GSE49569) from Vitis vinifera L. cv. Pinot noir clone Pommard (grafted to 101-14 rootstock, and trained in a double guyot system with vertically positioned shoots) described previously (Gouthu et al., 2014). In our study, we used expression data of 28,137 genes derived from pulp tissue of grape berries Collected at midvéraison (when $50 \%$ of the berries in the cluster had changed 
color) representing four developmental stages: the prevéraison (PV) (before the ripening initiation), green-soft (GS), pink-soft (PS), and red-soft (RS). The ripening features of the berries are summarized in Supplementary Table 1.

In this data, 186 NimbleGeneIDs lacked mapping to $12 \mathrm{X} v 1$ geneIDs, and in 13 instances two NimbleGeneIDs mapped to single $12 \mathrm{X} v 1$ geneID. These inconsistencies are a result of differences between the the genome annotation used for desigining the NimbleGen expression array and $12 \mathrm{X}$ v1 genome annotation. The 186 irrelavent older gene models were excluded and in case of the 13 duplicate instances, we chose one NimbleGeneIDs out of two per gene for further analysis, based on its highest differential expression observed across the four developmental stages. We averaged the expression values from three replicates. Moderated paired $t$-tests were used to compare the mean log expression intensities between the berry classes; GS compared with PV, PS compared with GS, RS compared with PS and RS compared with GS. The R/Bioconductor Limma package (Benjamini and Hochberg, 1995; Smyth, 2004) was used to calculate fold change in the transcript level of a given gene between two stages as described previously (Gouthu et al., 2014). This nonredundant dataset (see Supplementary Table 2), representing the fold change in the expression of 27,949 genes during developmental transitions of grape berry, was uploaded and analyzed using OMICs-Viewer tool as described previously (Paley and Karp, 2006; Dharmawardhana et al., 2013; Monaco et al., 2013).

\section{RESULTS}

\section{VitisCyc AS A RESOURCE FOR PLANT BIOLOGISTS}

Currently, VitisCyc harbors 68 super pathways, 362 biosynthesis pathways, 118 catabolic pathways, 5 detoxification pathways, 36 energy related pathways and 6 transport pathways, 10,908 enzymes, 2912 enzymatic reactions, 31 transport reactions and 2024 compounds. Users can access VitisCyc online from the webcite http://pathways.cgrb.oregonstate.edu. The VitisCyc entry page provides a summary of the database, serves as a springboard to facilitate search and browsing, and provides links to a cellular overview diagram, and the OMICs-Viewer tool (Figure 1). Searches can be carried out using the "Search" link in the menu on the top of the page, which may require selecting the species "Vitis vinifera." The browsing of the genes, enzymes, compounds, and pathways are supported using a framework of ontology based hierarchical classification schema. By using the hyperlinks, users can navigate up to the detailed pathway pages and views associated with each metabolite, gene ID, enzyme activity, reaction, and literature citations (Figure 2). The pathway page allows users to customize the levels of details shown on the page. The most detailed pathway view displays the structures of the metabolites. Whenever applicable, in the pathway page experimentally verified enzymatic activities or gene functions are depicted in bold. By clicking on the species comparison icon (Figure 2, on right side) users can compare a given pathway with reference databases, MetaCyc (Caspi et al., 2014), PlantCyc (Chae et al., 2012), and EcoCyc (Karp and Caspi, 2011), and/or across two or more species-specific, plant pathways databases (Figure 2) accessible from http://pathways.cgrb.oregonstate.edu. An example of detailed gene page is shown in Supplementary Figure 1. For extended help, users are encouraged to view online video tutorials on Cyc databases available at http://biocyc.org/webinar.shtml.

\section{MANUAL CURATION OF VitisCyc}

As described in the Methods, we projected a grape metabolic network based on extensive computational analyses, quality control steps, and manual curation. Manual curation includes (i) confirmation of computational mappings of genes to reactions and pathways, (ii) addition of grapevine-specific pathways, reactions, metabolites and biochemicals, and (iii) editing of reactions, sub-pathways, pathways and super-pathways including assignment of genes to a given enzyme and relevant pathways based on published grape literature. Examples of the manually curated pathways include four pathways of gibberellins and gibberellin precursors biosynthesis (Mattivi et al., 2006; He et al., 2010; Giacomelli et al., 2013); ten pathways involved in biosynthesis of five experimentally identified anthocyanins (cyanidin, delphinidin, peonidin, petunidin, malvidin and pelargonidin), and their 3-O-monoglycosides, and acyl-glycosides derivatives (Mattivi et al., 2006; He et al., 2010). We have also curated biosynthesis pathways and all associated entities for experimentally known flavonols in grapes, such as myricetin, quercetin, laricitrin, kaempferol, isorhamnetin and syringentin (Mattivi et al., 2006). Changes were also made in the biosynthesis pathways of plant hormones (e.g., abscisic acid, ethylene biosynthesis, jasmonic acid); trans-lycopene; pro-anthocyanidins; and isoflavonoids. In the generic interest of grape research community, we will continue to host pathways that can be useful in analyzing the data from other Vitis species, even though experimental evidence suggest their absence in Vitis vinifera.

Based on the published literature from $V$. vinifera, we entered 50 compounds, created 70 reactions, $\sim 20$ sub-pathways, assigned or edited function of $\sim 150$ genes associated with 80 reactions and deleted 10 subpathways. We also added 82 grape-specific literature references. Out of $\sim 2024$ metabolic compounds and biochemicals in VitisCyc, we have verified existence of about $\sim 400$ (20\%) entities by mining the published literature.

\section{UPLOAD, DISPLAY AND ANALYSIS OF USER-DEFINED EXPRESSION DATA}

The OMICs-Viewer tool, available from the VitisCyc entry page (Figure 1) or from the "Tools" menu available from the top navigation bar, enables users to upload and analyze a wide array of expression data including transcriptomes, proteomes, metabolomes, reaction flux, and/or any other data that can be assigned to metabolic genes, proteins, compounds, or reactions. The microaaray expression data (Supplementary Table 2) representing fold change in the expression of 27,949 unique genes in the pulp tissue of grape berries during four developmental transitions; in GS stage compared to PV; in PS stage compared to GS; in RS stage compared to PS, and in RS stage compared to GS was uploaded on OMICs-Viewer. We were able to map 7,566 out of total 27,949 genes to VitisCyc metabolic network. The remaining genes encode for transcription factors, regulatory proteins, structural proteins and for proteins of unknown function which are not yet associated to any metabolic reactions or pathways. Figure 3 shows relative change in the expression of grape genes 


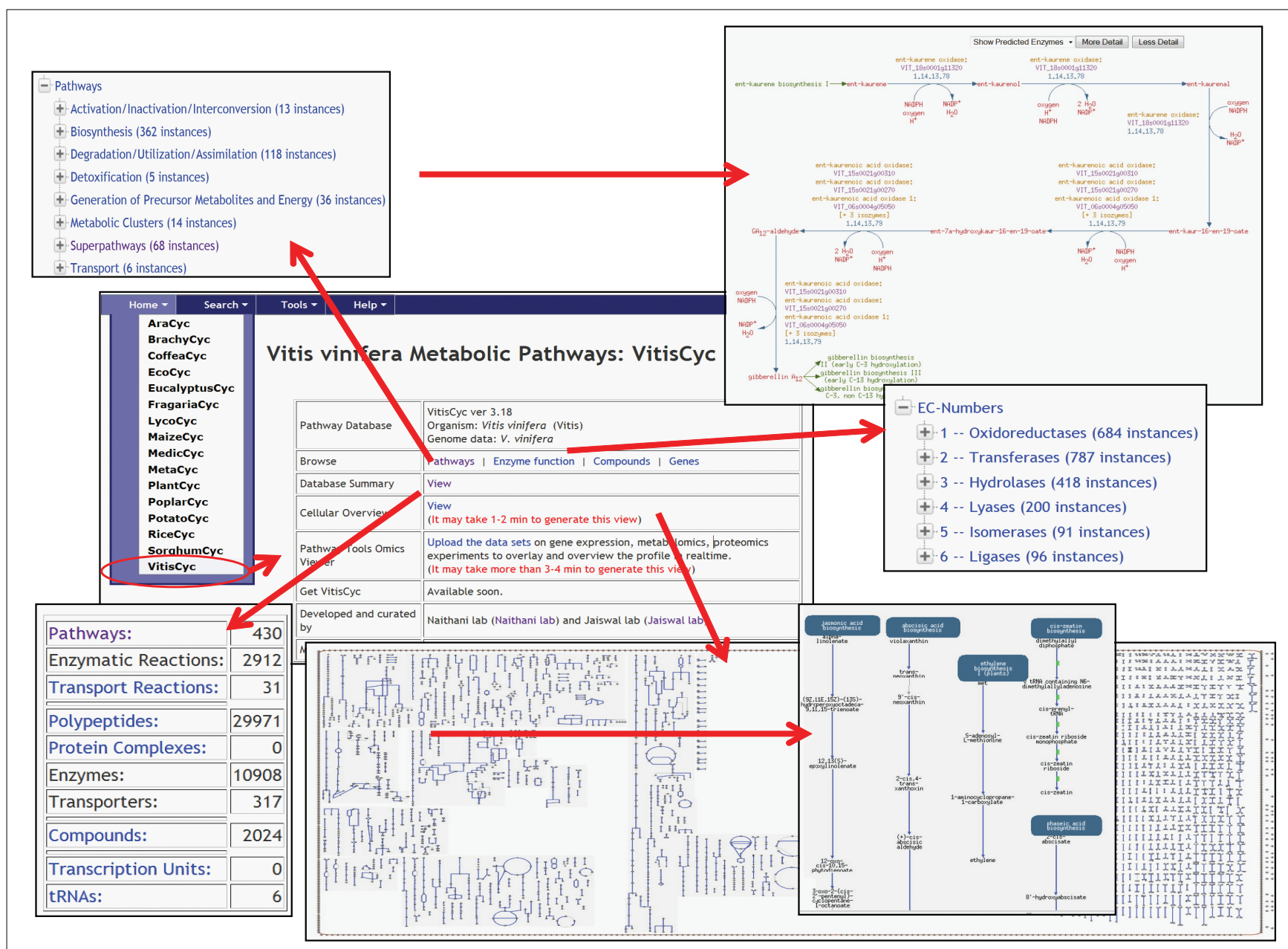

FIGURE 1 | A view of VitisCyc home page listing examples of various functions and utilities; a brief database summary, browsing of enzymes, and pathways using ontology based hierarchical classification scheme, view of detailed pathway page and cellular overview diagram.

during ripening transitions. The red color lines represent highest change in expression followed by yellow, dark blue, light blue and green in the cellular overview diagram.

We observed major changes at the cellular transcriptome during PV to GS transition that affects a wide array of genes associated with both primary and secondary metabolisms, hormone metabolism, photosynthesis, and energy pathways (Figure 3A). In contrast, the subsequent developmental transition from GS to PS mostly affects genes involved in secondary metabolic pathways (Figure 3B). During PS to RS transition, the change in the expression of genes of primary metabolic pathways and energy pathways can be observed in spite of major changes occurring in the plant hormone and secondary metabolic pathways (Figure 3C). The change in the expression of genes in the RS stage compared to GS is shown in Figure 3D.

Taking advantage of the OMICs-Viewer's interactive environment, we compiled a list of differentially expressed genes coding for enzymes of flavonol, flavonoid, anthocyanin, terpene, polysaccharides and plant hormone pathways (Supplementary Table 3). Clicking on a genes or a reaction on cellular overview diagram allowed us to survey various pathway pages. Figure 4 displays minimal view of the superpathway of flavonol, flavonoid, anthocyanins biosynthesis with the expression profile of few genes. By clicking on the "More Detail" icon on this pathway page, additional information, such as enzyme names with EC numbers and gene annotations can be displayed (see Supplementary Figure 2). A quick glance at the list and detailed pathway pages show that gene duplicates or members of a gene family carrying the same functional annotations, and thus mapped to the same reaction, do not share identical expression profiles (Figure 4, Supplementary Table 3). The change in the gene expression observed here represents only pulp tissue. It is likely that dramatic changes in the expression profile of the genes of this particular pathway could be observed in the skin or seed of grapes during the ripening transition as suggested by previous studies (Davies et al., 2010).

A majority of genes associated with various pathways (Supplementary Table 3) show differential expression during one or more ripening stage transitions, although transcription of the individual genes may peak either at early or late stage. Phytohormones control a variety of developmental processes in plants including fruit development and ripening (White, 2002). Therefore, we surveyed changes in the transcription of genes 


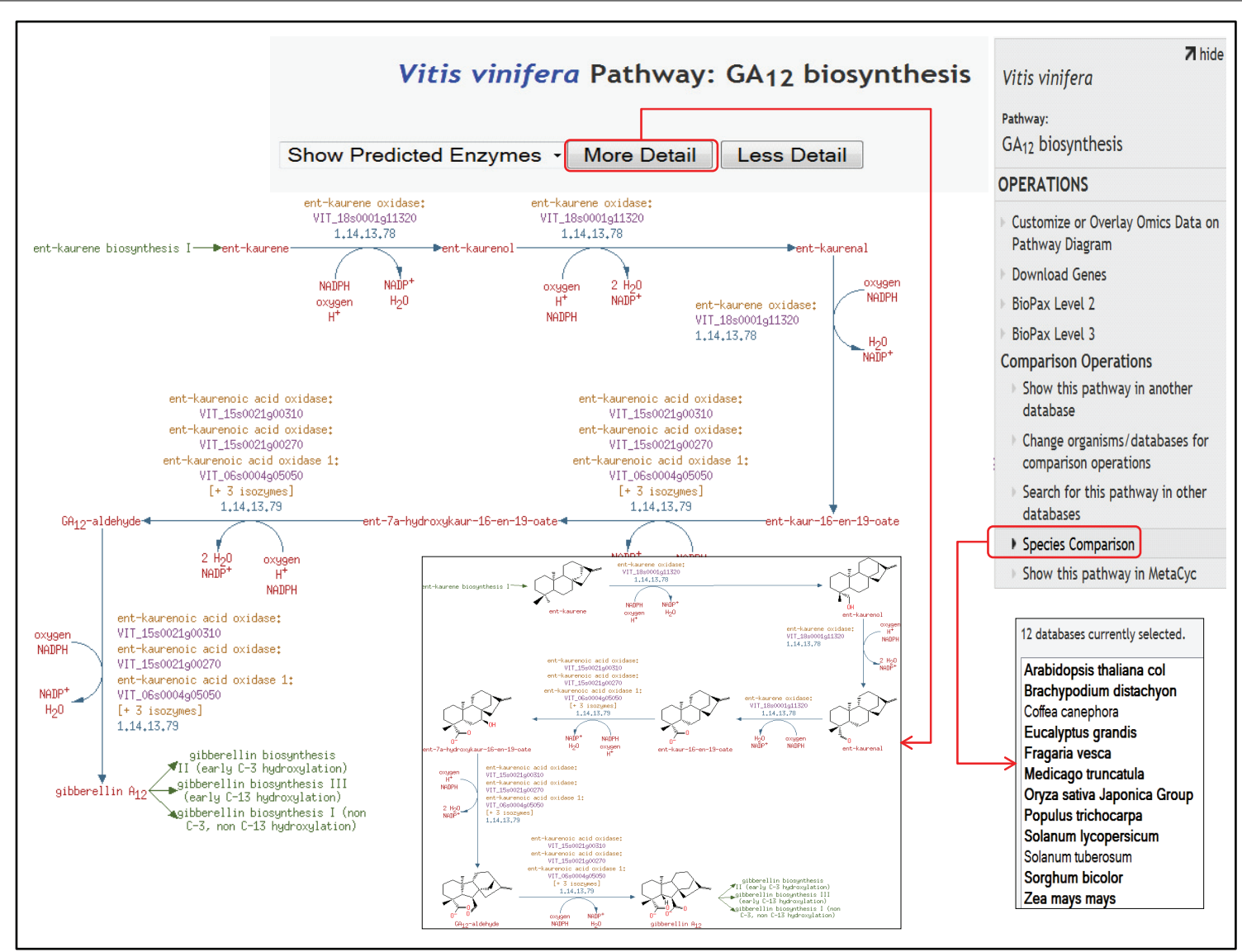

FIGURE 2 | A view of detailed pathway page showing GA12 biosynthesis pathway. The pathway page provides three layers of granularity including the most detailed format displaying structures of metabolites and biochemical. The GA12 biosynthesis pathway also shows links highlighted in green color for the preceding ent-kaurene biosynthesis pathway I, as well as for the successive gibberellin biosynthesis pathway I and III that use GA12 as substrate. On the right side, species comparison icon is highlighted that allows users to make comparisons of pathways across other publicly available, species-specific plant pathway databases; AraCyc (Zhang et al., 2005), MaizeCyc (Monaco et al., 2013), MedicCyc (Urbanczyk-Wochniak and Sumner, 2007), PoplarCyc (Zhang et al., 2010), RiceCyc (Dharmawardhana et al., 2013), LycoCyc, CoffeaCyc, PotatoCyc, BrachyCyc, SorghumCyc, FragariaCyc (Shulaev et al., 2011), and EucalyptusCyc, accessible from the website http://pathways.cgrb.oregonstate.edu. associated with the plant hormones metabolism (Supplementary Table 3 ). We found that 10 out of 13 genes of ethylene biosynthesis pathway show increased expression during PV to GS transition (ranging from 2 to $\sim 15$ fold). During GS to PS transition, the transcript's level of 9 out of 13 genes slightly decline, while the transcript's level of 4 genes remain more or less stable. In contrast, during PS to RS transition 9 out of 13 genes show increase and 4 genes show slight decline in transcription. These observations are consistent with the previous reports showing rise in the ethylene level before the ripening onset (Chervin et al., 2004; Sun et al., 2010) and increase in the transcripts level of ethylene biosynthesis genes before véraison (Ziliotto et al., 2012; Bottcher et al., 2013). Abscisic Acid is considered as the ripening promoter of grape berry and transiently increases during véraison (Deluc et al., 2009; Wheeler et al., 2009). We observed that 8 genes associated with Abscisic acid biosynthesis are upregulated ( 2 to $\sim 86$ fold-change) during PV to GS transition and then slightly decline in the subsequent stages except VIT_02s0087g00930 coding for 9-cis-epoxycarotenoid dioxygenase 2 (Supplementary Table 3).
Incidentally, the three genes encoding for ABA 8'-hydroxylase, involved in abscisic acid breakdown, exhibit sharp decline in transcription during PV to GS transition and continue to decline with ripening progress (Supplementary Table 3). Interestingly, 17 out of 30 genes involved in jasmonic acid biosynthesis show 2-5 folds increase, while 13 biosynthesis genes show decline in transcription during PV to GS transition (Supplementary Table 3). However, in subsequent ripening transitions, the transcript levels of the majority of jasmonic acid biosynthesis genes either show decline or remain stable. The function of gibberellin in berry development is mostly confined to the early stages of fruit formation and the berries do not maintain high levels of gibberellic acids (GAs) during the ripening (Giacomelli et al., 2013). The transcripts levels of most genes associated with gibberellin biosynthesis pathways decline during the berry ripening (Supplementary Table 3). Similarly, brassinosteroids are known to promote accumulation of pigments in grape (Symons et al., 2006). All three differentially expressed brassinosteroid biosynthesis genes show decrease in transcription during ripening. 


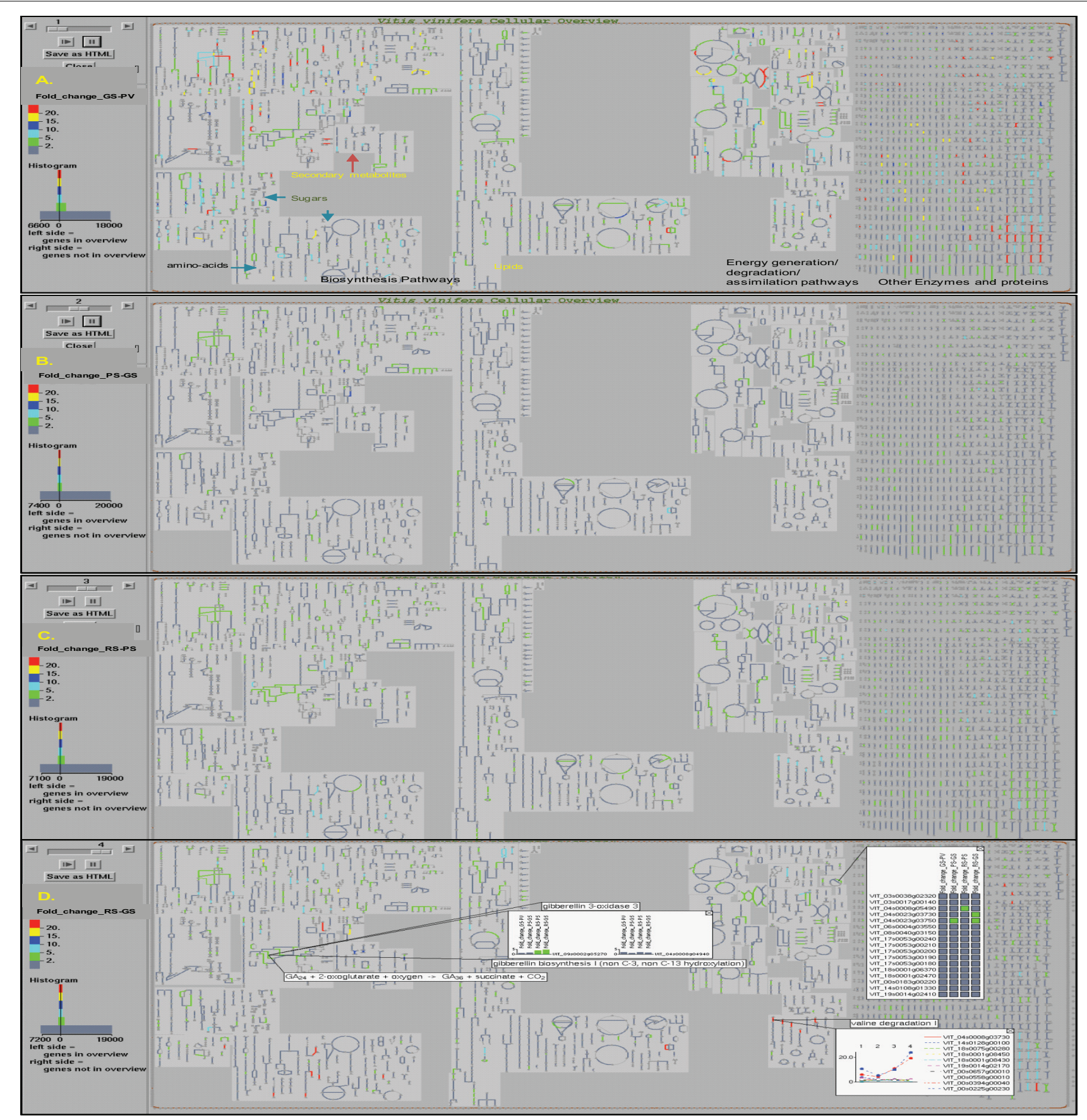

FIGURE 3 | The Vitis vinifera cellular overview diagram overlaid with expression data shows changes in the overall cellular gene expression profile in the pulp tissue of grape berry during ripening transitions observed in (A) green soft (GS) stage in comparison to prevéraison (PV) stage; (B) pink soft (PS) stage in comparison to GS stage; (C) red soft (RS) stage in comparison to PS stage; and (D) RS stage in comparison to early GS stage. The change in the gene expression values during ripening transitions are depicted by colors, red (>20 fold), yellow (15-20 fold), dark blue (10-15 fold), light blue (5-10 fold) and green ( $2-5$ fold). The examples of information accessible to users from the interactive cellular overview platform on reactions, pathways, compounds, and expression profiles of genes are shown (D). More details can be accessed by clicking on any entity and data can be displayed on pathways.
Overall, the down-regulation of gibberellin and brassinosteroid biosynthesis related transcripts and the upregulation of ethylene and $\mathrm{ABA}$ biosynthesis related transcripts suggest coordinated action of these hormones during the onset of grape berry ripening.

\section{DISCUSSION}

VitisCyc aims to provide a comprehensive view of grapevinespecific cellular metabolic networks, and a framework for analysis of systems level OMICs expression data. VitisCyc was constructed using Pathway Tools software (Karp et al., 2002) by integrating 


\section{Vitis vinifera: Phenylpropanoid Derivatives Biosynthesis}

Superpathway of flavonoid and flavonol biosynthesis leading to anthocyanin biosynthesis and accumulation

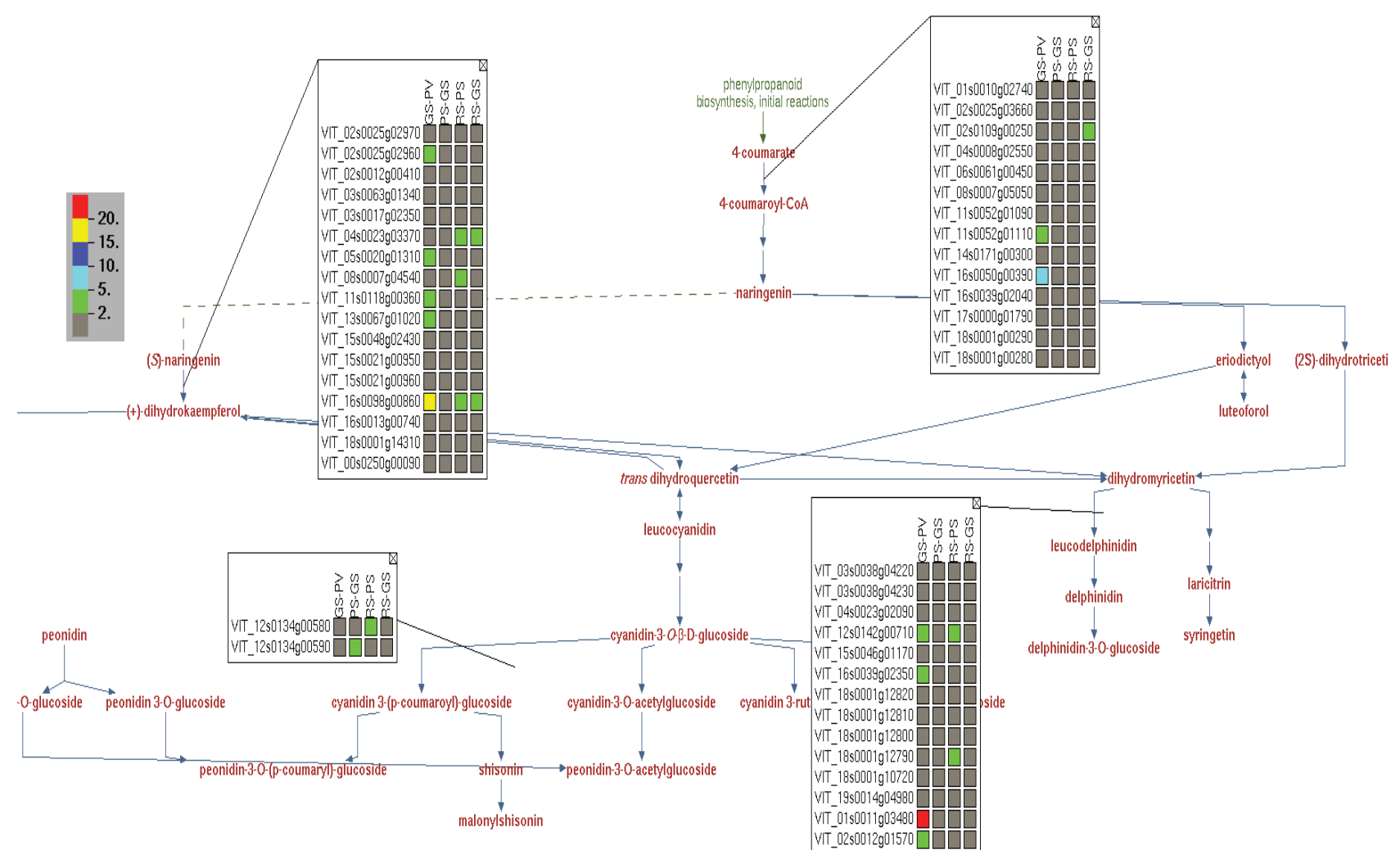

FIGURE 4 | A view of superpathway of flavonoid and flavonol biosynthesis leading to anthocyanin biosynthesis and accumulation showing expression profile of genes associated with individual reactions. The genes expression data depicts change in the expression of individual genes in the pulp tissue of grape berry during four ripening transitions. GS-PV: fold change in gene expression in green soft stage compared to prevéraison stage. PS-GS: fold change in gene expression in pink soft stage compared to green soft stage. RS-PS: fold change in gene expression in red soft, ripe stage compared to pink soft stage. The fold change in the gene expression values are depicted by red ( $>20)$, yellow (15-20), dark blue (10-15), light blue (5-10) and green (2-5) colors. By clicking on "More Detail" icon users can see functional annotations of the genes and chemical structure of the compounds. publicly available knowledge of grape metabolism with an in silico metabolic networks model based on the Vitis vinifera sequenced genome. The grape genome was sequenced first at $8 \mathrm{X}$ coverage (Jaillon et al., 2007), that was followed by release of genome annotation versions 12X v0 (Adam-Blondon et al., 2011) and 12X v1(Grimplet et al., 2012). Thus, users struggle with three genome annotations, back and forth to relate their findings with published studies, and publicly available data. In VitisCyc, we use the most updated $12 \mathrm{X}$ v1 annotations available from CRIBI (http://genomes.cribi.unipd.it/grape/), and also provide the gene annotation from $8 \mathrm{X}$ and $12 \mathrm{X}$ v0 annotations, if the gene model has not changed (Supplementary Figure 1). In the future, we would be able to incorporate new versions of genome annotation data as and when it becomes available. Our ability to map different geneIDs for existing gene models with incremental updates to gene annotations, pathways, reactions, and compounds (small molecules) allows users to build correspondence with the previously published research more easily.

The OMICs-Viewer, a built in tool (Paley and Karp, 2006) within VitisCyc, allows researchers to upload data of their choice, to paint it over the cellular overview diagram, and analyze expression of genes in context of a pathway and overall cellular metabolic network. Previously, we have shown analyses of microarray expression data and RNA-Seq data using the OMICsViewer tool in RiceCyc (Dharmawardhana et al., 2013) and MaizeCyc (Monaco et al., 2013), respectively. In this study, we used a subset of data, generated by Gouthu et al. (2014) that comprises gene expression data from pulp tissue of grape berries of four ripening stages: Green Hard, prevéraison stage (PV), Green Soft (GS), Pink Soft (PS), and Red Soft (RS). We demonstrated how information linked to a compound, reaction and genes can be accessed (Figure 3D) from the cellular overview diagram, and gene expression profiles can be painted on the pathway page. Users can navigate back and forth between the cellular overview diagram and the detailed pathway pages to pick differentially expressed pathways and genes. In addition to examples shown here, users can define maximum cut-off values, choose zero-centered or 1-centered scale to extract desired information, generate a table of individual pathways and associated genes exceeding the defined threshold, and/or choose to 
display data values on the genomic map. Data from multiple treatments/samples can also be uploaded simultaneously to generate animations of cellular expression profiles across samples.

Gouthu et al. (2014) have previously identified $\sim 3000$ differentially expressed genes by analyzing the skin, pulp, and seed tissues from Green Hard prevéraison (PV), Green Soft (GS), Pink Soft (PS), and Red Soft (RS) grape berries collected from mid-véraison cluster (when color change was visible in $50 \%$ of the berries). Their study was focused on asynchronous ripening between grape berries within the same cluster and suggested that in spite of the difference in the pace of ripening, the overall transcriptional program is similar between early and late ripening berries (Gouthu et al., 2014). Using OMICs-Viewer tool, we are able to extract additional information that was not garnered in the previous study. Our analysis suggests that the PV to GS transition triggers major changes in the overall cellular transcription activity leading to berry ripening and changes in the cellular metabolic profile (Figure 3). Mapping expression data on pathways allowed us not only to identify key reactions within various metabolic pathways that are up- and/or down regulated during ripening transitions, but also to account for the specific contribution of individual homologs mapped to the same reaction (Supplementary Table 3). Plant genomes harbor extensive gene duplications and large gene families (Shiu and Bleecker, 2003; Jaillon et al., 2007; Velasco et al., 2007; Myburg et al., 2014; Renny-Byfield and Wendel, 2014). The duplicate genes or members of a gene family carry the same functional annotations and thus map to the same reactions in the network unless an alternate function has been documented. Our analysis showed that often gene-duplicates do not share identical expression profiles and thus, are likely to be under the control of different regulators. Such information is specially useful in identification of candidate genes that are expressed in a specific cell- or tissue-type, at a particular stage of development, and in response to the changes in the plant's immediate environment. Users can also identify genes with redundant function and compare overlaps in expression profiles of genes associated with different reactions of a given pathway.

In addition, our analysis shows interplay between several hormones that is required to enable the fruit to transition from berry maturation to the ripening phase (Mcatee et al., 2013; Osorio et al., 2013), which is especially important to understand ripening process in non-climacteric fruits, including grape. We obsereved decline of gibberellins and brassinosteroids and up-regulation of ABA and ethylene during the ripening transitions, especially at GS compared to PV (Supplementary Table 3). Ethylene is known as a major ripening regulator in climacteric fruits, but it is not considered a major regulator in non-climacteric fruits. However, our data identified that $75 \%$ of ethylene biosynthesis genes were upregulated during PV to GS transition and therefore, supports role of ethylene in grape berry ripening, which is recently emerging (Ziliotto et al., 2012; Bottcher et al., 2013). Our data suggests that jasmonic acid is likely to play a role in the early onset of grape berry ripening (Supplementary Table 3). Knowledge about the role of jasmonic acid in grape berry is slowly emerging (Kondo and Fukuda, 2001; Fortes et al., 2011) and recent reports suggest its involvement in the ripening of another non-climacteric fruit strawberry (Concha et al., 2013; Preuss et al., 2014), and also in ethylene independent induction of lycopene biosynthesis in tomato (Liu et al., 2012). Overall, the finely tuned sampling of ripening stages, the extensive manual curation of the hormonerelated genes performed by our group, and the data visualization capacity of the OMICs-Viewer tools provide a clearer picture of the action of these hormones in the context of the grape metabolic network.

We view publicly available experimental data as a vital resource for improvement of the functional annotation of the grapevine genes and curation of enzymes, reactions, pathways, and compounds in the VitisCyc databases. Manual curation is an ongoing process and the growth of VitisCyc is expected as more data becomes available and integrated. Many grape researchers rely on another publicly available grape database VitisNet (Grimplet et al., 2012) and MapMan (Carbonell-Bejerano et al., 2013) for expression data analysis. VitisNet was built with CellDesigner platform and allows users to download one or more molecular networks from VitisNet and analyze their data using Cytoscape (Grimplet et al., 2009, 2012). We will be looking for opportunities to coordinate curation efforts especially on signaling pathways and small molecules together with the resources like MapMan (Ramsak et al., 2014), VitisNet and Plant Reactome (Monaco et al., 2013). We will also focus on efforts for developing automated scripts capable of extracting information from research articles with increased precision in order to provide more adequate information needed for OMICs data analysis, and the descriptions associated with biological function of genes/enzymes. We envision that in future, we could also provide a link to the phenotype database and to a metabolic mutant phenotype.

We expect that VitisCyc will aid grape researchers in discovering the broader role of new and known genes and/or molecular interactions in context of plant development, cells, tissues and organ differentiation. It could help in understanding how a plant's environment and genetic diversity relate to yields and the quality of its fruit's taste, aroma, flavor, and texture, and how the plant's overall metabolism and its ability to adapt is affected in response to climate change.

The VitisCyc is freely accessible online at http://pathways. cgrb.oregonstate.edu. The data content in the VitisCyc will be maintained and updated twice a year by continuously surveying the public resources and research articles as long as we have some support for curation and afterwards an archive copy will be maintained.

\section{ACKNOWLEDGMENTS}

Sushma Naithani and Pankaj Jaiswal conceived the project. Sushma Naithani, Pankaj Jaiswal, and Rajani Raja constructed the VitisCyc, Sushma Naithani supervised overall curation process and Sushma Naithani and Elijah N. Waddell did curations. Justin Elser carried out the Inparanoid-based annotations and provided technical support and software updates. Sushma Naithani, Satyanarayana Gouthu, and Laurent G. Deluc analyzed microarray data. Sushma Naithani, Pankaj Jaiswal, Satyanarayana Gouthu, Elijah N. Waddell and Laurent G. Deluc contributed in writing and editing the of the manuscript. We are grateful to Peter Karp, from SRI for excellent Pathway Tools support. This work 
was supported by the start up funds provided to Sushma Naithani and Pankaj Jaiswal by Oregon State University. Help from undergraduate student Kate Threlfall is acknowledged in the curation process.

\section{SUPPLEMENTARY MATERIAL}

The Supplementary Material for this article can be found online at: http://www.frontiersin.org/journal/10.3389/fpls.2014. 00644/abstract

\section{REFERENCES}

Adam-Blondon, A. F., Jaillon, O., Vezzulli, S., Zharkikh, A., Troggio, M., and Velasco, R. (2011). "Genome Sequence Initiatives," in Genetics, Genomics, and Breeding of Grapes, eds A. F. Adam-Blondon, J. M. Martinez-Zapater, and C. Kole (Enfield: Science Publishers and CRC Press), 211-234.

Benjamini, Y., and Hochberg, Y. (1995). Controlling the false discovery rate a practical and powerful approach to multiple testing. J. R. Statis. Soc. Ser. B-Methodol. 57, 289-300.

Bottcher, C., Burbidge, C. A., Boss, P. K., and Davies, C. (2013). Interactions between ethylene and auxin are crucial to the control of grape (Vitis vinifera L.) berry ripening. BMC Plant Biol. 13:222. doi: 10.1186/1471-2229-13-222

Carbonell-Bejerano, P., Santa Maria, E., Torres-Perez, R., Royo, C., Lijavetzky, D., Bravo, G., et al. (2013). Thermotolerance responses in ripening berries of Vitis vinifera L. cv muscat hamburg. Plant Cell Physiol. 54, 1200-1216. doi: 10.1093/pcp/pct071

Caspi, R., Altman, T., Billington, R., Dreher, K., Foerster, H., Fulcher, C. A., et al. (2014). The MetaCyc database of metabolic pathways and enzymes and the BioCyc collection of Pathway/Genome databases. Nucleic Acids Res. 42, D459-D471. doi: 10.1093/nar/gkt1103

Chae, L., Lee, I., Shin, J., and Rhee, S. Y. (2012). Towards understanding how molecular networks evolve in plants. Curr. Opin. Plant Biol. 15, 177-184. doi: 10.1016/j.pbi.2012.01.006

Chervin, C., El-Kereamy, A., Roustan, J. P., Latche, A., Lamon, J., and Bouzayen, M. (2004). Ethylene seems required for the berry development and ripening in grape, a non-climacteric fruit. Plant Sci. 167, 1301-1305. doi: 10.1016/J.Plantsci.2004.06.026

Chervin, C., Tira-Umphon, A., Terrier, N., Zouine, M., Severac, D., and Roustan, J. P. (2008). Stimulation of the grape berry expansion by ethylene and effects on related gene transcripts, over the ripening phase. Physiol. Plant. 134, 534-546. doi: 10.1111/j.1399-3054.2008.01158.x

Concha, C. M., Figueroa, N. E., Poblete, L. A., Onate, F. A., Schwab, W., and Figueroa, C. R. (2013). Methyl jasmonate treatment induces changes in fruit ripening by modifying the expression of several ripening genes in Fragaria chiloensis fruit. Plant Physiol. Biochem. 70, 433-444. doi: 10.1016/j.plaphy.2013.06.008

Costantini, L., Battilana, J., Lamaj, F., Fanizza, G., and Grando, M. S. (2008). Berry and phenology-related traits in grapevine (Vitis vinifera L.): from quantitative trait loci to underlying genes. BMC Plant Biol. 8:38. doi: 10.1186/14712229-8-38

Cramer, G. R., Ergul, A., Grimplet, J., Tillett, R. L., Tattersall, E. A., Bohlman, M. C., et al. (2007). Water and salinity stress in grapevines: early and late changes in transcript and metabolite profiles. Funct. Integr. Genomics 7, 111-134. doi: 10.1007/s10142-006-0039-y

Dai, Z. W., Leon, C., Feil, R., Lunn, J. E., Delrot, S., and Gomes, E. (2013). Metabolic profiling reveals coordinated switches in primary carbohydrate metabolism in grape berry (Vitis vinifera L.), a non-climacteric fleshy fruit. J. Exp. Bot. 64, 1345-1355. doi: 10.1093/jxb/ers396

Da Silva, F. G., Iandolino, A., Al-Kayal, F., Bohlman, M. C., Cushman, M. A., Lim, H., et al. (2005). Characterizing the grape transcriptome: analysis of ESTs from multiple Vitis species and development of a compendium of gene expression during berry development. Plant Physiol. 139, 574-597. doi: 10.1104/pp.105. 065748

Davies, H. V., Shepherd, L. V., Stewart, D., Frank, T., Rohlig, R. M., and Engel, K. H. (2010). Metabolome variability in crop plant species-when, where, how much and so what? Regul. Toxicol. Pharmacol. 58, S54-S61. doi: 10.1016/j.yrtph.2010.07.004
De, L. J., Besse, S., and Boucher, F. (2010). Diet and heart health: moderate wine drinking strengthens the cardioprotective effects of fish consumption. Curr. Pharm. Biotechnol. 11, 911-921. doi: 10.2174/138920110793262024

Deluc, L. G., Grimplet, J., Wheatley, M. D., Tillett, R. L., Quilici, D. R., Osborne, C., et al. (2007). Transcriptomic and metabolite analyses of Cabernet Sauvignon grape berry development. BMC Genomics 8:429. doi: 10.1186/1471-2164-8-429

Deluc, L. G., Quilici, D. R., Decendit, A., Grimplet, J., Wheatley, M. D., Schlauch, K. A., et al. (2009). Water deficit alters differentially metabolic pathways affecting important flavor and quality traits in grape berries of cabernet sauvignon and chardonnay. BMC Genomics 10:212. doi: 10.1186/1471-2164-10-212

Dharmawardhana, P., Ren, L., Amarasinghe, V., Monaco, M. K., Thomason, J., Ravenscroft, D., et al. (2013). A genome scale metabolic network for rice and accompanying analysis of tryptophan, auxin and serotonin biosynthesis regulation under biotic stress. Rice 6, 1-15. doi: 10.1186/1939-8433-6-15

Doligez, A., Bouquet, A., Danglot, Y., Lahogue, F., Riaz, S., Meredith, P., et al. (2002). Genetic mapping of grapevine (Vitis vinifera L.) applied to the detection of QTLs for seedlessness and berry weight. Theor. Appl. Genet. 105, 780-795. doi: 10.1007/s00122-002-0951-z

Espinoza, C., Vega, A., Medina, C., Schlauch, K., Cramer, G., and Arce-Johnson, P. (2006). Gene expression associated with compatible viral diseases in grapevine cultivars. Funct. Integr. Genomics 7, 95-110. doi: 10.1007/s10142-006-0031-6

Fernandez, L., Torregrosa, L., Terrier, N., Sreekantan, L., Grimplet, J., Davies, C., et al. (2007). Identification of genes associated with flesh morphogenesis during grapevine fruit development. Plant Mol. Biol. 63, 307-323. doi: 10.1007/s11103006-9090-2

Figueiredo, A., Fortes, A. M., Ferreira, S., Sebastiana, M., Choi, Y. H., Sousa, L., et al. (2008). Transcriptional and metabolic profiling of grape (Vitis vinifera L.) leaves unravel possible innate resistance against pathogenic fungi. J. Exp. Bot. 59, 3371-3381. doi: 10.1093/jxb/ern187

Fischer, B. M., Salakhutdinov, I., Akkurt, M., Eibach, R., Edwards, K. J., Topfer, R., et al. (2004). Quantitative trait locus analysis of fungal disease resistance factors on a molecular map of grapevine. Theor. Appl. Genet. 108, 501-515. doi: 10.1007/s00122-003-1445-3

Fortes, A. M., Agudelo-Romero, P., Silva, M. S., Ali, K., Sousa, L., Maltese, F., et al. (2011). Transcript and metabolite analysis in trincadeira cultivar reveals novel information regarding the dynamics of grape ripening. BMC Plant Biol. 11:149. doi: 10.1186/1471-2229-11-149

Gatto, P., Vrhovsek, U., Muth, J., Segala, C., Romualdi, C., Fontana, P., et al. (2008). Ripening and genotype control stilbene accumulation in healthy grapes. J. Agric. Food Chem. 56, 11773-11785. doi: 10.1021/jf8017707

Giacomelli, L., Rota-Stabelli, O., Masuero, D., Acheampong, A. K., Moretto, M., Caputi, L., et al. (2013). Gibberellin metabolism in Vitis vinifera L. during bloom and fruit-set: functional characterization and evolution of grapevine gibberellin oxidases. J. Exp. Bot. 64, 4403-4419. doi: 10.1093/jxb/ert251

Giribaldi, M., Perugini, I., Sauvage, F. X., and Schubert, A. (2007). Analysis of protein changes during grape berry ripening by 2-DE and MALDI-TOF. Proteomics 7, 3154-3170. doi: 10.1002/pmic.200600974

Gouthu, S., O’neil, S. T., Di, Y., Ansarolia, M., Megraw, M., and Deluc, L. G. (2014). A comparative study of ripening among berries of the grape cluster reveals an altered transcriptional programme and enhanced ripening rate in delayed berries. J. Exp. Bot. 65, 5889-5902. doi: 10.1093/jxb/eru329

Grimplet, J., Cramer, G. R., Dickerson, J. A., Mathiason, K., Van Hemert, J., and Fennell, A. Y. (2009). VitisNet: "Omics" integration through grapevine molecular networks. PLoS ONE 4:e8365. doi: 10.1371/journal.pone.0008365

Grimplet, J., Deluc, L. G., Tillett, R. L., Wheatley, M. D., Schlauch, K. A., Cramer, G. R., et al. (2007). Tissue-specific mRNA expression profiling in grape berry tissues. BMC Genomics 8:187. doi: 10.1186/1471-2164-8-187

Grimplet, J., Van Hemert, J., Carbonell-Bejerano, P., Diaz-Riquelme, J., Dickerson, J., Fennell, A., et al. (2012). Comparative analysis of grapevine whole-genome gene predictions, functional annotation, categorization and integration of the predicted gene sequences. BMC Res. Notes 5:213. doi: 10.1186/1756-0500-5-213

He, F., Mu, L., Yan, G. L., Liang, N. N., Pan, Q. H., Wang, J., et al. (2010). Biosynthesis of anthocyanins and their regulation in colored grapes. Molecules 15, 9057-9091. doi: 10.3390/molecules 15129057

Jaillon, O., Aury, J. M., Noel, B., Policriti, A., Clepet, C., Casagrande, A., et al. (2007). The grapevine genome sequence suggests ancestral hexaploidization in major angiosperm phyla. Nature 449, 463-467. doi: 10.1038/nature06148

Karp, P. D., and Caspi, R. (2011). A survey of metabolic databases emphasizing the MetaCyc family. Arch. Toxicol. 85, 1015-1033. doi: 10.1007/s00204-011-0705-2 
Karp, P. D., Paley, S., and Altman, T. (2013). Data mining in the MetaCyc family of pathway databases. Methods Mol. Biol. 939, 183-200. doi: 10.1007/978-1-62703107-3_12

Karp, P. D., Paley, S., and Romero, P. (2002). The pathway tools software. Bioinformatics 18(Suppl. 1), S225-S232. doi: 10.1093/bioinformatics/18.suppl_1.S225

Kondo, S., and Fukuda, K. (2001). Changes of jasmonates in grape berries and their possible roles in fruit development. Sci. Hortic. 91, 275-288. doi: 10.1016/S03044238(01)00271-0

Liu, L., Wei, J., Zhang, M., Zhang, L., Li, C., and Wang, Q. (2012). Ethylene independent induction of lycopene biosynthesis in tomato fruits by jasmonates. J. Exp. Bot. 63, 5751-5761. doi: 10.1093/jxb/ers224

Lund, S. T., Peng, F. Y., Nayar, T., Reid, K. E., and Schlosser, J. (2008). Gene expression analyses in individual grape (Vitis vinifera L.) berries during ripening initiation reveal that pigmentation intensity is a valid indicator of developmental staging within the cluster. Plant Mol. Biol. 68, 301-315. doi: 10.1007/s11103008-9371-z

Martínez-Esteso, M. J., Vilella-Antón, M. T., Pedreño, M. Á., Valero, M. L., and Bru-Martínez, R. (2013). iTRAQ-based protein profiling provides insights into the central metabolism changes driving grape berry development and ripening. BMC Plant Biol. 13:167. doi: 10.1186/1471-2229-13-167

Martinez-Esteso, M. J., Selles-Marchart, S., Lijavetzky, D., Pedreno, M. A., and Bru-Martinez, R. (2011). A DIGE-based quantitative proteomic analysis of grape berry flesh development and ripening reveals key events in sugar and organic acid metabolism. J. Exp. Bot. 62, 2521-2569. doi: 10.1093/jxb/ erq434

Mathiason, K., He, D., Grimplet, J., Venkateswari, J., Galbraith, D. W., Or, E., et al. (2009). Transcript profiling in Vitis riparia during chilling requirement fulfillment reveals coordination of gene expression patterns with optimized bud break. Funct. Integr. Genomics 9, 81-96. doi: 10.1007/s10142-0080090-y

Mattivi, F., Guzzon, R., Vrhovsek, U., Stefanini, M., and Velasco, R. (2006). Metabolite profiling of grape: flavonols and anthocyanins. J. Agric. Food Chem. 54, 7692-7702. doi: 10.1021/jf061538c

Mcatee, P., Karim, S., Schaffer, R., and David, K. (2013). A dynamic interplay between phytohormones is required for fruit development, maturation, and ripening. Front. Plant Sci. 4:79. doi: 10.3389/fpls.2013.00079

Monaco, M. K., Sen, T. Z., Dharmawardhana, P. D., Ren, L., Schaeffer, M., Naithani, S., et al. (2013). Maize metabolic network construction and transcriptome analysis. Plant Genome 6, 1-12. doi: 10.3835/Plantgenome2012.09.0025

Myburg, A. A., Grattapaglia, D., Tuskan, G. A., Hellsten, U., Hayes, R. D., Grimwood, J., et al. (2014). The genome of eucalyptus grandis. Nature 510, 356-362. doi: 10.1038/Nature13308

Myles, S., Boyko, A. R., Owens, C. L., Brown, P. J., Grassi, F., Aradhya, M. K., et al. (2011). Genetic structure and domestication history of the grape. Proc. Natl. Acad. Sci. U.S.A. 108, 3530-3535. doi: 10.1073/pnas.1009363108

Osorio, S., Scossa, F., and Fernie, A. R. (2013). Molecular regulation of fruit ripening. Front. Plant Sci. 4:198. doi: 10.3389/fpls.2013.00198

Paley, S. M., and Karp, P. D. (2006). The pathway tools cellular overview diagram and omics viewer. Nucleic Acids Res. 34, 3771-3778. doi: 10.1093/nar/gkl334

Pilati, S., Perazzolli, M., Malossini, A., Cestaro, A., Dematte, L., Fontana, P., et al. (2007). Genome-wide transcriptional analysis of grapevine berry ripening reveals a set of genes similarly modulated during three seasons and the occurrence of an oxidative burst at veraison. BMC Genomics 8:428. doi: 10.1186/1471-2164-8-428

Preuss, A., Augustin, C., Figueroa, C. R., Hoffmann, T., Valpuesta, V., Sevilla, J. F., et al. (2014). Expression of a functional jasmonic acid carboxyl methyltransferase is negatively correlated with strawberry fruit development. J. Plant Physiol. 171, 1315-1324. doi: 10.1016/j.jplph.2014.06.004

Ramsak, Z., Baebler, S., Rotter, A., Korbar, M., Mozetic, I., Usadel, B., et al. (2014). GoMapMan: integration, consolidation and visualization of plant gene annotations within the MapMan ontology. Nucleic Acids Res. 42, D1167-D1175. doi: 10.1093/Nar/Gkt1056

Renny-Byfield, S., and Wendel, J. F. (2014). Doubling down on genomes: polyploidy and crop plants. Am. J. Bot. 101, 1711-1725. doi: 10.3732/ajb.1400119

Salmaso, M., Malacarne, G., Troggio, M., Faes, G., Stefanini, M., Grando, M. S., et al. (2008). A grapevine (Vitis vinifera L.) genetic map integrating the position of 139 expressed genes. Theor Appl. Genet. 116, 1129-1143. doi: 10.1007/s00122008-0741-3
Sarry, J. E., Sommerer, N., Sauvage, F. X., Bergoin, A., Rossignol, M., Albagnac, G., et al. (2004). Grape berry biochemistry revisited upon proteomic analysis of the mesocarp. Proteomics 4, 201-215. doi: 10.1002/Pmic.200300499

Shiu, S. H., and Bleecker, A. B. (2003). Expansion of the receptor-like kinase/Pelle gene family and receptor-like proteins in Arabidopsis. Plant Physiol. 132, 530-543. doi: 10.1104/pp.103.021964

Shulaev, V., Sargent, D. J., Crowhurst, R. N., Mockler, T. C., Folkerts, O., Delcher, A. L., et al. (2011). The genome of woodland strawberry (Fragaria vesca). Nat. Genet. 43, 109-116. doi: 10.1038/ng.740

Smoliga, J. M., Baur, J. A., and Hausenblas, H. A. (2011). Resveratrol and healtha comprehensive review of human clinical trials. Mol. Nutr. Food Res. 55, 1129-1141. doi: 10.1002/mnfr.201100143

Smyth, G. K. (2004). Linear models and empirical bayes methods for assessing differential expression in microarray experiments. Stat. Appl. Genet. Mol. Biol. 3, Article3. doi: 10.2202/1544-6115.1027

Sun, L., Zhang, M., Ren, J., Qi, J., Zhang, G., and Leng, P. (2010). Reciprocity between abscisic acid and ethylene at the onset of berry ripening and after harvest. BMC Plant Biol. 10:257. doi: 10.1186/1471-2229-10-257

Symons, G. M., Davies, C., Shavrukov, Y., Dry, I. B., Reid, J. B., and Thomas, M. R. (2006). Grapes on steroids. Brassinosteroids are involved in grape berry ripening. Plant Physiol. 140, 150-158. doi: 10.1104/pp.105.070706

Tattersall, E. A., Grimplet, J., Deluc, L., Wheatley, M. D., Vincent, D., Osborne, C., et al. (2007). Transcript abundance profiles reveal larger and more complex responses of grapevine to chilling compared to osmotic and salinity stress. Funct. Integr. Genomics 7, 317-333. doi: 10.1007/s10142-007-0051-x

Terrier, N., Glissant, D., Grimplet, J., Barrieu, F., Abbal, P., Couture, C., et al. (2005). Isogene specific oligo arrays reveal multifaceted changes in gene expression during grape berry (Vitis vinifera L.) development. Planta 222, 832-847. doi: 10.1007/s00425-005-0017-y

Urbanczyk-Wochniak, E., and Sumner, L. W. (2007). MedicCyc: a biochemical pathway database for Medicago truncatula. Bioinformatics 23, 1418-1423. doi: 10.1093/bioinformatics/btm040

Velasco, R., Zharkikh, A., Troggio, M., Cartwright, D. A., Cestaro, A., Pruss, D., et al. (2007). A high quality draft consensus sequence of the genome of a heterozygous grapevine variety. PLoS ONE 2:e1326. doi: 10.1371/journal.pone.0001326

Vezzulli, S., Troggio, M., Coppola, G., Jermakow, A., Cartwright, D., Zharkikh, A., et al. (2008). A reference integrated map for cultivated grapevine (Vitis vinifera L.) from three crosses, based on 283 SSR and 501 SNP-based markers. Theor. Appl. Genet. 117, 499-511. doi: 10.1007/s00122-008-0794-3

Waters, D. L. E., Holton, T. A., Ablett, E. M., Slade Lee, L., and Henry, R. J. (2006). The ripening wine grape berry skin transcriptome. Plant Sci. 171, 132-138. doi: 10.1016/j.plantsci.2006.03.002

Waters, D. L., Holton, T. A., Ablett, E. M., Lee, L. S., and Henry, R. J. (2005). cDNA microarray analysis of developing grape (Vitis vinifera cv. Shiraz) berry skin. Funct. Integr. Genomics 5, 40-58. doi: 10.1007/s10142-004-0124-z

Wheeler, S., Loveys, B., Ford, C., and Davies, C. (2009). The relationship between the expression of abscisic acid biosynthesis genes, accumulation of abscisic acid and the promotion of Vitis vinifera L. berry ripening by abscisic acid. Aust. J. Grape Wine Res. 15, 195-204. doi: 10.1111/j.1755-0238.2008.00045.x

White, P. J. (2002). Recent advances in fruit development and ripening: an overview. J. Exp. Bot. 53, 1995-2000. doi: 10.1093/jxb/erf105

Wu, J. M., and Hsieh, T. C. (2011). Resveratrol: a cardioprotective substance. Ann. N.Y. Acad. Sci. 1215, 16-21. doi: 10.1111/j.1749-6632.2010.05854.x

Zamboni, A., Di Carli, M., Guzzo, F., Stocchero, M., Zenoni, S., Ferrarini, A., et al. (2010). Identification of putative stage-specific grapevine berry biomarkers and omics data integration into networks. Plant Physiol. 154, 1439-1459. doi: 10.1104/pp.110.160275

Zenoni, S., Ferrarini, A., Giacomelli, E., Xumerle, L., Fasoli, M., Malerba, G., et al. (2010). Characterization of transcriptional complexity during berry development in Vitis vinifera using RNA-Seq. Plant Physiol. 152, 1787-1795. doi: 10.1104/pp.109.149716

Zhang, P., Dreher, K., Karthikeyan, A., Chi, A., Pujar, A., Caspi, R., et al. (2010). Creation of a genome-wide metabolic pathway database for Populus trichocarpa using a new approach for reconstruction and curation of metabolic pathways for plants. Plant Physiol. 153, 1479-1491. doi: 10.1104/pp.110. 157396

Zhang, P., Foerster, H., Tissier, C. P., Mueller, L., Paley, S., Karp, P. D., et al. (2005). MetaCyc and AraCyc. Metabolic pathway databases for plant research. Plant Physiol. 138, 27-37. doi: 10.1104/pp.105.060376 
Ziliotto, F., Corso, M., Rizzini, F. M., Rasori, A., Botton, A., and Bonghi, C. (2012). Grape berry ripening delay induced by a pre-veraison NAA treatment is paralleled by a shift in the expression pattern of auxin- and ethylene-related genes. BMC Plant Biol. 12:185. doi: 10.1186/1471-2229-12-185

Conflict of Interest Statement: The Guest Associate Editor Mario Pezzotti declares that, despite having collaborated with author Laurent G. Deluc, the review process was handled objectively and no conflict of interest exists. The author Laurent G. Deluc declares that, despite having collaborated with the editor Mario Pezzotti, the review process was handled objectively and no conflict of interest exists. The authors declare that the research was conducted in the absence of any commercial or financial relationships that could be construed as a potential conflict of interest.
Received: 23 August 2014; accepted: 01 November 2014; published online: 09 December 2014.

Citation: Naithani S, Raja R, Waddell EN, Elser J, Gouthu S, Deluc LG and Jaiswal P (2014) VitisCyc: a metabolic pathway knowledgebase for grapevine (Vitis vinifera). Front. Plant Sci. 5:644. doi: 10.3389/fpls.2014.00644

This article was submitted to Crop Science and Horticulture, a section of the journal Frontiers in Plant Science.

Copyright (c) 2014 Naithani, Raja, Waddell, Elser, Gouthu, Deluc and Jaiswal. This is an open-access article distributed under the terms of the Creative Commons Attribution License (CC BY). The use, distribution or reproduction in other forums is permitted, provided the original author(s) or licensor are credited and that the original publication in this journal is cited, in accordance with accepted academic practice. No use, distribution or reproduction is permitted which does not comply with these terms. 